\title{
Design of New Architecture for Model Management Systems using Knowledge Sharing Concept
}

\author{
Sandeep Kautish \\ Research Scholar \\ Bhagwant University, Ajmer Rajasthan
}

\author{
M.P. Thapliyal, PhD. \\ Professor Deptt. Of Computer Engg. \\ H.N.B. Garhwal University, Srinagar Uttrakhand
}

\begin{abstract}
Information system designing is no more typical process with the advent of various methods and techniques. Decision Support Systems are one class of Information Systems which helps decision makers in situations when problem is multifaceted and complex enough to understand and solve manually. The most critical part of Decision Support Systems designing is developing Model Management Systems, a systems which determine the feasibility of one decision model and makes comparison of same with its alternatives to suggest best suitable decision model for given problem. Structured modeling is the oldest and widely accepted approach for MMS designing and this paper explores this oldest approach of MMS development and aligns the concept of knowledge sharing with Structured Modeling for designing more effective Model Management systems. Finally a new architecture is proposed for MMS designing with integration of knowledge sharing concept.
\end{abstract}

\section{Keywords}

Model Management Systems (MMS), Information Systems, Structured Modeling.

\section{INTRODUCTION}

Decision (DSS) can be explained as a class of information systems which is computerized and support the activities of decision-making. According to Turban [1], Decision Support System is "an interactive, flexible, and adaptable computerbased information system, developed especially for better decision making as it supports the solution of a non-structured management problem. It utilizes data which provides an easyto-use interface, and allows for the decision maker's own insights". It's impossible to give a more precise definition with all the DSS facets, although the above definition is also good, we can say that these are context- and task-specific. One fact that is suitable about DSS is, it includes a decisionmaking process, and this definition is widely acceptable by all.

Decision Support Systems has several components such as Database Management Systems (DBMS), Model Based Management Systems (MMS) and a User Interface. Out of these three components, designing of the MMS is the most typical one. There are many approaches being used for designing Model Management Systems. Structured modeling is the oldest and widely accepted approach. Improvement in productivity of models has been an issue of concern for a long time. Research which explores the modeling process can be traced to early 60's (e.g., Harary, Norman and Cartwright [9] and results are found as areas such as structural modeling, cybernetics and simulation. Recently, rapid advances in computer and decision support technologies have focused much attention on applying these technologies to develop a productive modeling environment. The resulting computer aided modeling systems are called model management systems (MMS).

The architecture of a typical DSS model is presented here Figure 1 depicts a typical architecture which is based upon expert system.

Figure 1 - Typical architecture of DSS

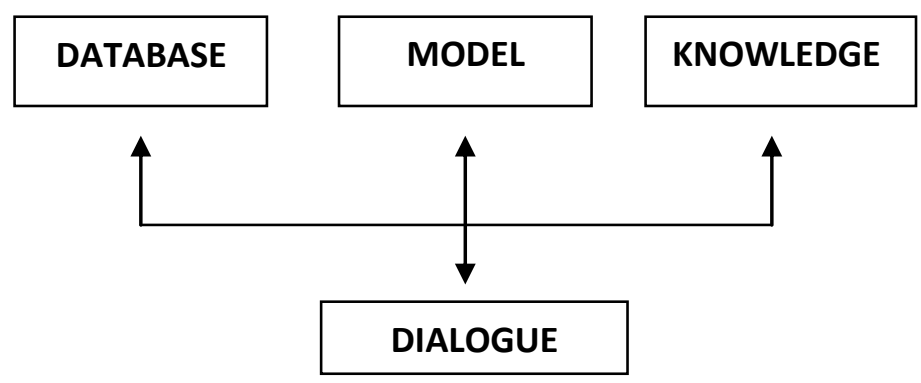

The architecture consists of four main components: a database, a model management subsystem, dialogue system and knowledge management subsystem.

\section{CONCEPT OF KNOWLEDGE SHARING}

The roots of knowledge sharing concepts lie within the technology transfer and innovation literature. The main focus is on the successes of failures of different nations' in fostering the economic growth through the development of technology. Whereas some of the theorists argues that national innovation and growth rates are due to high investments in physical and human capital [3, 4], 'assimilation theorists' on the other hand says that entrepreneurship, effective learning, and innovation are separate but act equally important variables that affect to the development.. An understanding of the importance of sharing of ideas is the central of both the approaches. The firms are mastering and getting into new practices like product design, manufacturing processes, and organizational designs which are the result of successful knowledge sharing [5]. On technology transfer it is the recent volume by the title of Richard Nelson's, Technology, Learning \& Innovation [16], it is seen that knowledge sharing is occurring through dynamic leaning process where customer and the supplier can continually been interacted by the organizations in order to innovate or imitate creativity. The case of technology transfer which was proposed by Lall [13] is been considered technologies being used in industries are mainly obtained from the industrialized world by the developing countries, and their major problem is technology and to master, adapt and improve knowledge and unlike the sale of a good, when the physical delivery takes place than only the transaction is complete, a prolonged process is a successful transfer of the technology, and the completion of transaction involves local 
learning.

As per the conclusions the development experts have led to recommend the activities focused on facilitating knowledge sharing and rather on transmitting Northern Knowledge to the South are more successful $[8,3]$. We can say that knowledge communication is important; knowledge sharing is a process that determines whether the organizational learning occurred and therefore it's the success of knowledge sharing.

In information systems, individual-level knowledge sharing studies have been conducted, organizational behavior, strategic management, and psychology but no systematic review has been conducted till now. Previous reviews have limited their focused on technical issues related to knowledge sharing or knowledge transfer across functional units, organizations, or within inter organization networks [17].

\section{REDEFINING THE ROLE OF KNOWLEDGE SHARING}

Emphasizing on the role of knowledge sharing in designing Model Management Systems (MMS) is one of the major objectives of this paper. Research on DSS has increased since past decade but very few research attempts are made on inclusion of knowledge sharing concepts. Structured modeling [11] was the theory which focused on functional relationship among the models during modeling process. Structure modeling considers each model of model base as single entity. The objective of Structured Modeling is to provide a mathematical and computer-based framework for creating, representing, and manipulating a wide variety of models. The framework consists of a hierarchical and acyclic graph which is organized, partitioned, and attributed to represent the mathematical structure of a model. The computer-based environment facilitates immediate expression evaluation, optimizing and solving problems. Subject to successful implementation, Structured Modeling enables decision makers to work on variety of decision models with greater productivity. In addition, its acceptance by non specialists will result into more developments in the field of technology. Moreover, it will cross-fertilize several distinct areas of management science i.e. operations research, artificial intelligence and software engineering.

Geoffrion's [7] work is the base of graph based approach presented here. The only difference between framework presented here and structuring modeling is the latter focuses on functional relationships among different models and consider high level of cohesion among these models. The proposed framework deals not only with representation of model, method of integrating and selecting models.

Structured modeling is totally different from any one of the traditional modeling paradigms. It has wide expressive power to represent models and model classes from a variety of different paradigms. In fact it can also represent many paradigms themselves. This facilitates integration across modeling paradigms which supports knowledge sharing between different modeling paradigms as and when required [19]. Integration across different modeling paradigms within a single discipline-specific modeling tradition usually reduces the complexity of decision models and allows decision makers to utilize best possible alternative models.
Structured modeling is good enough to encompass a variety of paradigms from a variety of modeling traditions. For example, structured modeling accommodates the relational and entityrelationship data model paradigms from database management and some versions of the semantic network paradigm from artificial intelligence [11]. As another example, structured modeling accommodates paradigms from accounting, finance, marketing, and other functional areas of business [18]. Therefore, we can say that Structured Modeling supports knowledge sharing among different decision models. Though Structured Modeling does not directly support solver integration at the algorithmic level. However, structured modeling can support solver integration in most aspects external to algorithmic. For example, integrated capabilities for answering ad hoc queries (of database management systems) and for doing definitional calculation (of spreadsheet programs) is an explicit requirement for a structured modeling system. The prototype system currently in development will achieve this when completed.

\section{ARCHITECTURE OF PROPOSED FRAMEWORK}

Since the approach used in the study is based on reasoning and judgment, a different Model base management system is proposed in the paper. The new architecture of model management system is presented which consists of 3 modules: a modeling subsystem, a modeling deployment subsystem and an inference engine.

The proposed framework includes two major subsystems: modeling and model deployment system out of which modeling system concentrates on improving effectiveness of models and model deployment subsystem focuses on proper utilization of models. Another important component of framework is inference engine which makes connectivity between model subsystem and model deployment subsystem. The model deployment subsystem accepts user queries, generates reports and provides assistance via consultation messages. The query processor is the part of the model deployment subsystem which provides interface between end user and the system. It interprets user's query in system specific commands. The report generator produces the output in user specified format.

The modeling subsystem works as model builder which builds useful models. There are three more functions of this subsystem i.e. knowledge acquisition, assisting users in model selection and integration. Another function is to apply artificial intelligence by using automatic modeling which is used to create new models or modify existing models using AI techniques [14].

The inference engine is the core part of this framework as it is one which controls and directs entire operations of the system. The user specific commands [16] are being interpreted and executed by inference engine. In addition, inference engine controls model base, fetch knowledge from knowledge base and performs inferences, whenever applicable. After obtaining information, inference engine passes this information to other subsystems and generates non availability report if information is not available. 
Figure 2: Knowledge based framework for MMS

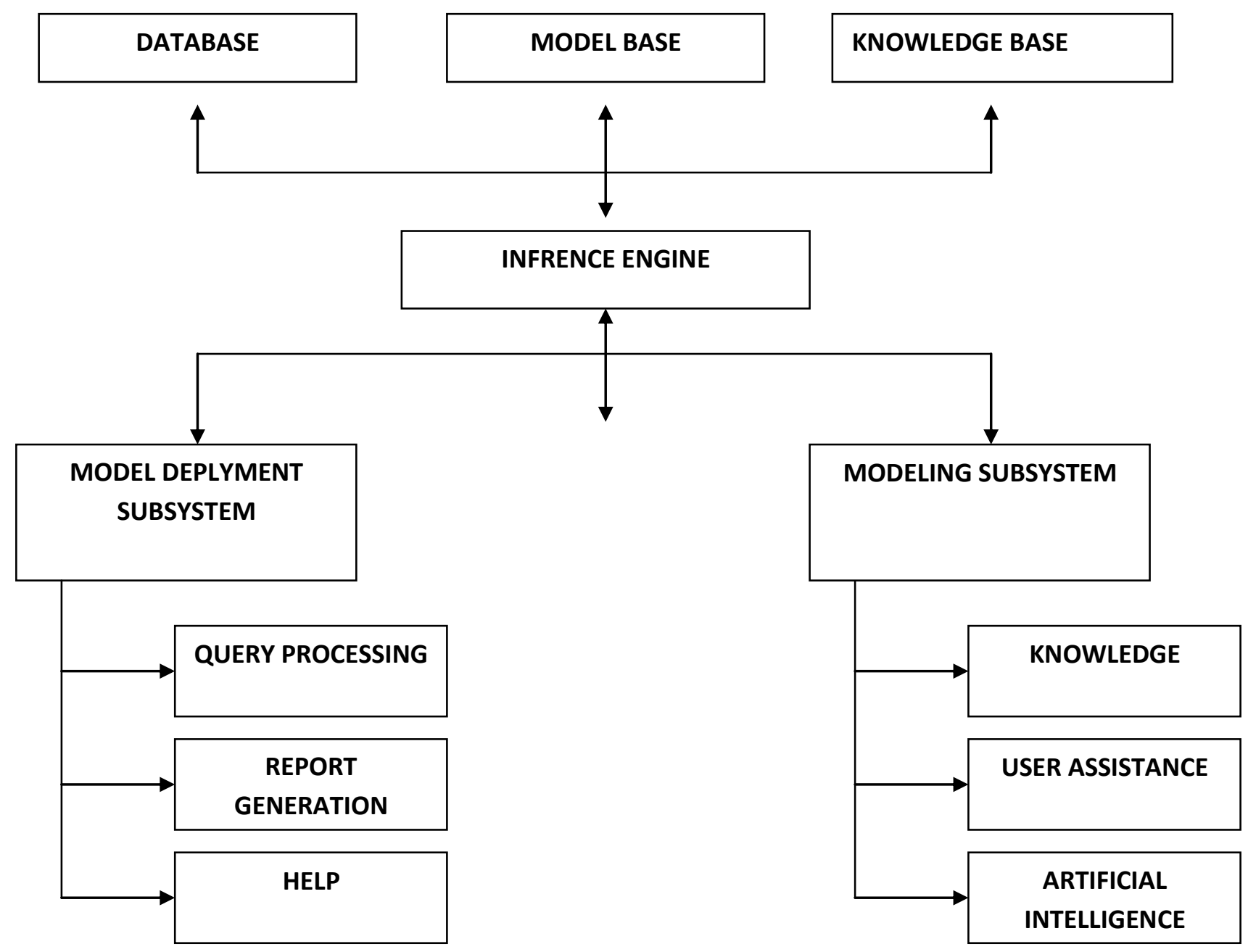

In against any user query, search will be made in database and will be retrieved if information available. Otherwise, the model database will be searched for seeking models which can produce desired information. If model available then there will be another check for its required inputs [14]. If no desired model is available, there will be attempt to develop composite model. This implementation will be based on graph theory.

\section{IMPLEMENTATION OF FRAMEWORK}

Previous section described the roadmap for developing knowledge based framework for model management system. This prototype requires to be integrated with one powerful query language i.e. SQL or Prolog for actual implementation. As this paper emphasizing on conceptual issues of the MMS designing, we are presenting basic outline of the prototype which demonstrate the feasibility of the proposed framework. It is an integrated framework with model selection and integration capability. The architecture of the prototype system is based on the architecture proposed in Figure 2. It has two major subsystems. First is the model utilization subsystem and second is modeling subsystem. A graph based inference engine facilitates the integration among models as well as model base and the database. The model utilization subsystem performs three major roles: processing queries, report generation and help utility. For this, we require an interactive and powerful query language which enables users to access both the database and model base without any need to know where the information is stored. The prototype system maintains a data dictionary to enable user in understanding various terms used in the process. In this query processing process, when a user specifies the category of required information such as "Production", the system will retrieve attributes related to the term from the data dictionary. For example, for the "production", the related attributes might be "product" or "year". The system will prompt for these values and accordingly will process the query. For example, the query for "production in 2012" might have syntax like this:

SELECT production

WHERE "PRODUCT="MILK" YEAR="2012"

\section{CONCLUSION}

This paper is an attempt to present unconventional framework for designing Model Management Systems (MMS) which supports knowledge sharing phenomena among different decision models. Section One of this paper covers the preliminary and fundamental issues of Decision Supports Systems (DSS) and Model Management Systems (MMS). 
Section Two introduces the concept of knowledge sharing and reveals the available literature on the topic. Section Three and Four are the core parts of the paper as it presents new architecture of designing MMS with integration of knowledge sharing feature into it and suggests a prototype model implementation of the proposed framework subsequently.

\section{REFERENCES}

[1] A. Efram Turbon (1985), Decision Support Systems and business intelligent systems" $8^{\text {th }}$ edition, Prentice Hall, $p$. 14.

[2] Argote, L. and Ingram, P. (2000). "Knowledge transfer: A basis for competitive advantage in firms," Organizational Behavior and Human Decision Processes, 82 (1): $150-169$.

[3] Eberhart, R. \& Kennedy, J. (1995). A new optimizer using particle swarm theory, Proceedings of the Sixth International Symposium on Micromachine and Human Science, Nagoya, Japan. p. 39-43, 1995.

[4] Efraim Turban, Jay E. Aronson, Ting-Peng Liang (2008). Decision Support Systems and Intelligent Systems. p. 574

[5] Ellerman, D., Denning S. and Hanna, N. (2001). "Active learning and development assistance," Journal of Knowledge Management, 5, 2: 171- 179.

[6] F. Harary, rz Norman, and D. Cartwright, Structural Models: An Introduction to the Theory of Directed Graphs. New York: Wiley, 1965.

[7] Geoffrion, European Journal of Operational Research, 1985, vol. 21, issue 1, pages 113-120.

[8] Grant, R. M. (1996). "Toward a knowledge-based theory of the firm," Strategic Management Journal, 17: 109122.

[9] Harary, F., Norman, R.Z., and Cartwright, D. (1965), Structural Models: An Introduction to the Theory of Directed Graphs, New York: John Wiley \& Sons.

[10] Inkpen, A. C. and Dinur, A. (1998)."Knowledge management processes and international joint ventures," Organization Science, 9 (4): 454-468.
[11] Kim, J. I. and Lau, L. J. (1994). "The sources of economic growth in the East Asian newly industrialized countries," Journal of Japanese and International Economics, 18: 235-271. Tavel, P. 2007 Modeling and Simulation Design. AK Peters Ltd.

[12] Kim, L. and Nelson, R. R. (2000). Technology, learning, and innovation: Experiences of newly industrializing economies, Cambridge, UK: Cambridge University Press.

[13] Lall, S. (2000). "Technological change and industrialization in the Asian newly industrializing economies: Achievements and challenges," Chapter 2 in L. Kim and R.R. Nelson, editors, Technology, learning, and innovation: Experiences of newly industrializing economies, Cambridge, UK: Cambridge University Press.

[14] Liang, T. P. (1985), "Integrating Model Management with Data Management in Decision Support Systems," Decision Support Systems, 1:3, pp. 221-232.

[15] Moreau, E. (2006). The impact of intelligent decision support systems on intellectual task success: An empirical investigation, Decision Support Systems, n. 42, p. 593-607, 2006

[16] Nelson, R. and Rosenberg, N. (1993). "Technical innovation and national systems," chapter 1 in R.R. Nelson, R. R., editor, National innovation systems: A comparative analysis, New York: Oxford University Press.

[17] Oliveira, F. \& Lima Neto, F. (2008). An Evolutionary Approach to Provide Flexible Decision Dialogues in Intelligent Decision Support Systems, Proceedings of the 8th International Conference on Hybrid Intelligent Systems (HIS 2008), Barcelona, Spain, 2008.

[18] Prusak, L. (1999). "Action review of knowledge management: Report and recommendations," World Bank.

[19] Young, A. (1993). "The tyranny of numbers: Confronting the statistical realities of the East Asian growth experience," Quarterly Journal of Economics, 110: 641-680 